\title{
La gestion de l'environnement dans le Pacifique. Moyens et objectifs
}

Guy Agniel

\section{(2) OpenEdition}

1 Journals

Édition électronique

URL : http://journals.openedition.org/jso/3842

DOI : $10.4000 /$ jso.3842

ISSN : 1760-7256

Éditeur

Société des océanistes

\section{Édition imprimée}

Date de publication : 15 décembre 2008

Pagination : 145-151

ISBN : 978-2-85430-012-3

ISSN : 0300-953x

Référence électronique

Guy Agniel, "La gestion de l'environnement dans le Pacifique. Moyens et objectifs », Journal de la Société des Océanistes [En ligne], 126-127 | Année 2008, mis en ligne le 15 décembre 2011, consulté le 16 juin 2020. URL : http://journals.openedition.org/jso/3842 ; DOl : https://doi.org/10.4000/jso.3842 


\title{
La gestion de l'environnement dans le Pacifique. Moyens et objectifs
}

\author{
par
}

Guy AGNIEL*

\section{RÉSUMÉ}

La protection de l'environnement dans le Pacifique est une préoccupation relativement récente. Elle s'organise à partir des actions du Programme régional océanien de l'environnement (PROE), organisation régionale qui regroupe les États et territoires du Pacifique ainsi que les puissances occidentales qui ont des intérêts dans la région. Ses moyens, assez faibles, sont mobilisés par deux programmes principaux : le programme "écosystèmes insulaires" dont l'un des objectifs est la lutte contre les espèces bio-envahissantes et le programme "Avenirs océaniens » qui prend en compte la gestion de la pollution et des déchets et les changements climatiques.

Mots-CLÉS : Pacifique, environnement, déchets, espèces bio-envahissantes, changement climatique, polluant organique persistant

Le Pacifique a toujours fait rêver. On lui associe inconsciemment des plages de sable blanc immaculées et des lagons aux eaux limpides et colorées : c'est la Nature dans sa pureté originelle. Las! Si Stevenson et Gauguin ont peutêtre connu pareil paradis sur terre, l'irrésistible progression de la modernité et du progrès technique a porté quelque atteinte à son image. La lascive vahiné a subi bien souvent de la société de consommation l'irréparable outrage et pollue son environnement avec une efficacité digne de ses consœurs occidentales.

\begin{abstract}
Managing and protecting Pacific's Islands environment is quite a recent concern. It is organized around actions headed by the South Pacific Regional Environment Program (SPREP), a regional organisation which gathers, on one hand, Pacific's Islands States and Territories, and, on the other hand, Western States which have interests in the region. The SPREP ressources, which are, comparatively, not so important, are dedicated to two main Programs: the "Islands ecosystems Program», which includes struggling against bio-invasives species, and the "Oceanian Futures» Program, which aims to control the dumping of toxic wastes, pollution and climatic changes.
\end{abstract}

Keywords: Pacific, environment, waste, bio-invasives species, climatic change, persistant organic pollutant

Les pays et États insulaires ont souvent traité le sujet avec une certaine nonchalance et n'ont vraiment pris conscience de la gravité de la situation qu'assez récemment.

Or, le Pacifique se trouve au carrefour de toutes les problématiques de l'environnement et du développement durable, étant à la fois l'une des régions les plus riches du monde pour la diversité de son milieu, mais également l'une des plus vulnérables. Il s'est doté depuis peu d'une organisation internationale à vocation régionale, le Programme régional océanien de l'environ-

* Professeur, université de la Nouvelle-Calédonie, agniel@univ-nc.nc 
nement (PROE), qui se trouve confronté à de nombreux axes de lutte contre la pollution et de gestion de l'environnement, tous plus prioritaires les uns que les autres. Il n'est pas possible de les examiner tous, ni même d'en dresser la longue et fastidieuse liste.

Aussi nous limiterons-nous, après un bref exposé des moyens de lutte contre la dégradation des environnements naturels déjà mis en place, à quelques-uns des aspects les plus délicats de la protection de l'environnement dans le Pacifique.

\section{Le dispositif conventionnel régional}

Trois accords régionaux constituent l'architecture principale du dispositif actuel de protection de l'environnement :

1. La convention sur la protection de la nature dans le Pacifique Sud (dite aussi convention d'Apia, 1976). Les Parties à cette convention ont décidé de promouvoir la mise en œuvre d'aires protégées (par exemple : parc national ou réserve) afin de préserver des échantillons des écosystèmes naturels représentatifs en portant une attention toute particulière aux espèces menacées. Font aussi partie des sites protégés les paysages remarquables, les formations géologiques frappantes et les régions ou objets présentant un intérêt esthétique ou une valeur historique culturelle ou scientifique. Conclue à Apia (Samoa) le 12 juin 1976, elle est entrée en vigueur le 26 juin 1990.

2. La convention relative à la protection des ressources naturelles et de l'environnement de la région du Pacifique Sud (dite aussi convention de Nouméa, 1986). Les Parties se sont engagées à assurer par tous moyens la protection, la mise en valeur et la gestion du milieu marin et côtier de la zone d'application de la convention tout en reconnaissant la valeur économique et sociale des ressources naturelles du milieu ainsi que l'existence de traditions et de cultures propres aux peuples du Pacifique. Adoptée à Nouméa (Nouvelle-Calédonie) le 25 novembre 1986, elle est entrée en vigueur le 22 août 1990.

Cette convention a été mise en œuvre par les protocoles du même jour à savoir le protocole de coopération dans les interventions d'urgence contre les incidents générateurs de pollution dans la région du Pacifique Sud, et le protocole sur la prévention de la pollution de la région du Pacifique Sud résultant de l'immersion de déchets (ensemble 4 annexes).

3. La convention portant création du Programme régional océanien de l'environnement
(PROE). Adoptée à Apia le 16 juin 1993, elle est entrée en vigueur le 11 août 1996. C'est l'outil conventionnel le plus important, fruit des initiatives conjointes de la Communauté du Pacifique (ex-Commission du Pacifique Sud ou CPS), du Programme des Nations unies pour l'Environnement (PNUE), du Bureau de Coopération économique du Pacifique Sud (SPEC) et de la Commission économique et sociale des Nations unies pour l'Asie et le Pacifique (CESAP). Le PROE a pour objectif de promouvoir la coopération régionale en vue de protéger et d'améliorer l'environnement, tout en pérennisant le développement durable pour les générations futures. Les programmes d'action portent sur l'ensemble des problèmes qui se posent aux États et territoires insulaires du Pacifique, que nous allons voir ci-dessous.

\section{La conservation de la diversité biologique}

Conservation de la diversité biologique ou de la « biodiversité », elle vise à protéger les espèces endémiques à la région et à encourager l'utilisation écologique durable des ressources biologiques de la région. Dans le cadre de ce programme, a été approuvée la création de zones de conservation dans neuf États insulaires ; de plus, deux programmes ambitieux ont été lancés en 1994 : le programme régional pour la conservation des mammifères marins et la stratégie régionale de conservation de l'avifaune.

\section{Le changement global et l'élévation du niveau de la mer}

Il s'agit d'un programme de recherche et d'observation des phénomènes climatiques et physiques résultant de "l'effet de serre»: le réchauffement de l'atmosphère pourrait avoir pour conséquence une élévation du niveau de la mer menaçant l'existence d'États océaniens constitués par des atolls. Neuf États ou territoires non autonomes sont directement concernés par ce problème : Les îles Cook, les États fédérés de Micronésie, Guam, Kiribati, les Marshall, Palau, les Samoa, Tonga et Tuvalu.

\section{La gestion planifiée de l'environnement}

Cette gestion planifiée recouvre deux éléments : le programme d'assistance technique à l'environnement régional (ATER) et celui des stratégies nationales de gestion de l'environnement (SNAGE). Chaque pays élabore sa propre stratégie en fonction de sa situation économique, 
physique, culturelle et sociale et définit le programme d'actions prioritaires à entreprendre pour protéger son environnement, tandis que la Banque asiatique du développement (BAD) et le PNUD, en collaboration avec le Bureau australien d'assistance au développement international (BAADO) et l'Union mondiale pour la conservation de la nature (UICN), financent les programmes d'assistance technique.

\section{La gestion planifiée des zones côtières}

Les zones côtières jouent un rôle primordial car elles abritent la plus grande partie de la population et constituent le noyau du développement économique ; c'est également dans ces zones que se concentrent l'agriculture vivrière et la pêche commerciale. La dégradation rapide des habitats côtiers et la surexploitation des ressources naturelles inquiètent les gouvernements en raison des conflits de plus en plus fréquents concernant l'utilisation des ressources, en particulier dans les centres urbains caractérisés par une forte croissance démographique. Les îles de superficie restreinte, notamment les atolls, sont les plus particulièrement menacées.

Les interventions d'urgence contre les incidents générateurs de pollution et contre la pollution en général

Le PROE a mis en place un programme régional de prévention de la pollution et de gestion des déchets. Nous allons en présenter les objectifs spécifiques.

- La gestion des déchets sur terre, en eau douce, en mer et dans l'air. À ce titre la Papouasie-Nouvelle-Guinée est à l'origine d'un projet de convention visant à l'interdiction $d u$ transport de matières toxiques et dangereuses dans la zone Pacifique. Cette convention, dans le droit-fil du traité de Rarotonga de 1985 sur la zone dénucléarisée du Pacifique Sud, avait peu de chances d'être un jour effective en raison de l'existence d'États côtiers fortement industrialisés comme les États-Unis et le Japon. Cependant les travaux ont abouti à un projet de texte qui a été discuté et signé en 1995 : c'est le traité de Waigani (16 septembre 1995), signé par les membres du Forum du Pacifique. Il prévoit le contrôle des mouvements de déchets dangereux produits par les États membres et interdit les importations sur le territoire des États membres insulaires de déchets produits dans d'autres pays. On notera, qu'en application de ces dispositions, les îles Salomon ont été condamnées lors de la neuvième conférence du Mouvement pour un Pacifique indépendant et dénucléarisé (18-24 janvier 2003) pour les avoir enfreintes en acceptant de stocker les ordures ménagères de Taïwan sur l'île de Makira pour un profit à court terme. Les participants de cette conférence ont demandé à tous les gouvernements des îles du Pacifique d'honorer le traité en interdisant à tous les pays industrialisés de venir stocker leurs déchets dans le Pacifique.

- La prévention et le contrôle de la pollution de la terre, des eaux douces, des eaux côtières et des eaux de haute mer.

- L'élaboration d'une base de données régionale sur les sources, les niveaux, les quantités, les tendances et les effets des produits polluants.

- La sensibilisation aux problèmes de pollution, d'éducation et de formation.

Dans le cadre de l'éducation, l'information et la sensibilisation en matière d'environnement, le PROE s'est donné pour tâche de réaliser du matériel pédagogique (notamment un manuel d'enseignement sur l'environnement) et des programmes d'études destinés aux écoles et aux ministères de l'éducation.

Un des rôles du PROE est le suivi du sommet de la terre, essentiellement l'Agenda 21 et la déclaration de Rio. Désigné par les Nations unies comme l'organisation régionale apte à en coordonner les préparatifs, sa principale action a été la mise en place de la conférence de la Barbade qui a examiné et entériné un programme d'action pour le développement durable des petits États insulaires en voie de développement. En septembre 1995, l'agrément de Niue a permis d'atteindre le total des dix ratifications nécessaire à l'entrée en vigueur de la convention. Depuis sont venues s'y ajouter les ratifications des îles Marshall (2003), de Palau (2005), des États-Unis (2005) et du Vanuatu qui, en janvier 2006 a été le dernier des États signataires à accepter d'être liés par la convention.

\section{Les actions}

Le PROE a été confronté, dès l'origine, à toutes les formes possibles d'atteintes à l'environnement. Après une période de tâtonnements, il s'est néanmoins orienté vers les objectifs qui ont été identifiés comme prioritaires par ses membres. Aujourd'hui, l'action du PROE s'applique à deux programmes majeurs : celui concernant les écosystèmes insulaires et le programme Avenirs océaniens. Cependant, il convient de garder à 
l'esprit que l'organisation régionale ne dispose guère que d'un pouvoir de proposition renforcé par quelques mesures incitatives, notamment de soutien technique à des actions ciblées. La faiblesse de ses ressources financières (seuls quatre membres, dont deux extérieurs à la région, sont en mesure de participer de façon significative à son budget de fonctionnement ${ }^{1}$ ) la rend extrêmement dépendante des bailleurs de fonds internationaux ${ }^{2}$. Quant aux initiatives sur fonds propres elles sont pratiquement inexistantes. Les réalisations, quand elles sont menées à terme, paraissent souvent dérisoires à l'œil occidental, alors qu'elles constituent en général un progrès important compte tenu du contexte local.

Les programmes du PROE peuvent donc être perçus comme un inventaire d'actions soumises non à la bonne volonté de ses membres mais à leur capacité de réalisation. Des esprits chagrins y verront une psalmodie de formules incantatoires dissimulant une forme d'appel à financement par la communauté internationale. Prétendre l'inverse ne serait peut-être pas totalement conforme à la réalité. Mais nous nous trouvons dans un domaine, comme tant d'autres en droit, dans lequel la bonne foi se présume.

\section{Le programme relatif aux écosystèmes insulaires}

Son objectif principal est d'améliorer la gestion et la conservation des ressources naturelles tout en luttant contre l'appauvrissement de la biodiversité. Le point d'étape est fixé en 2010. Les dirigeants des États et territoires du Pacifique sont bien conscients que ce problème n'est pas nouveau : dans le Pacifique, plusieurs sociétés insulaires ont, à une époque ou à une autre, adopté des modes d'existence incompatibles avec le principe du développement durable ou bien ont été confrontées à des changements environnementaux externes. Certaines ont disparu, d'autres ont migré. Mais, même dans les sociétés qui ont d'instinct adopté un mode de vie respectueux de l'environnement terrestre et marin, leur développement naturel s'est accompagné d'importantes modifications de l'écosystème, voire de la disparition d'espèces. Ces changements se sont accélérés et amplifiés depuis cinquante ans, se traduisant par la dégradation des zones de captage des eaux, le déclin des forêts et de leur biodiversité, la surpêche, les dégâts des espèces envahissantes. Parallèlement, on cons- tate une surpopulation des zones côtières des petits États et territoires, phénomène qui s'accompagne d'un épuisement des ressources, de la disparition de la mangrove et d'une mauvaise gestion des déchets.

À l'initiative du gouvernement néo-zélandais, a été élaboré un programme de travail sur la biodiversité insulaire qui a très vite mis en lumière le déclin continu de celle-ci. L'objectif recherché a alors été de définir les principales mesures à prendre et leur financement. En effet, la presque totalité des États et territoires concernés ne disposent pas de ressources suffisantes et doivent se tourner vers les bailleurs de fonds internationaux. Cette incapacité à engager des actions d'envergure est durement illustrée par la mise en œuvre des mesures de soutien à la convention de Ramsar sur les zones humides (en Océanie, il s'agit essentiellement des mangroves, des récifs coralliens et des herbiers). Les parties océaniennes à cette Convention (les Marshall, Palau, la Papouasie Nouvelle-Guinée et les Samoa) ont des moyens financiers trop limités pour s'engager activement dans son application : l'association du PROE à la convention est là aussi plus symbolique qu'opérationnelle.

Mais l'aspect le plus important de ce programme est la prise de conscience de la nécessité de lutter contre les espèces envahissantes, qui sont considérées comme la plus grande des menaces pesant sur la biodiversité et le développement durable dans la région du Pacifique. Or, depuis les quinze dernières années, l'introduction intentionnelle ou accidentelle d'espèces non endémiques s'est accrue, au point que les bioinvasions du milieu marin, y compris par le vecteur des transports maritimes (eaux de ballast et salissures de coques) ont été jugées comme faisant partie des quatre plus grandes menaces pesant sur la biodiversité et les écosystèmes du monde. Le programme des Nations unies pour l'environnement (PNUE) et l'Union mondiale pour la nature (UICN) l'ont considéré, au sommet mondial du développement durable de Johannesburg (2002), comme la deuxième des grandes menaces après la disparition des habitats biologiques. Les milieux insulaires du Pacifique sont particulièrement vulnérables: quatre espèces déjà ont été identifiées dans la région, où elles sont devenues envahissantes ou sont en passe de l'être. Il s'agit de l'anatife Chtalamus proteus, de plusieurs espèces de macroalgues, d'espèces nui-

1. Ainsi, les contributions statutaires des membres pour 2008 sont estimées à un montant de $695000 €$ dont près de $69 \%$ sont fournis par l'Australie, les États-Unis, la France et la Nouvelle-Zélande, les participations respectives des vingt autres membres (Pitcairn étant dispensé) variant de $1,09 \%$ à $2,18 \%$.

2. Ces organismes, internationaux ou nationaux, contribuent aux financements du programme Écosystèmes insulaires et Avenirs océaniens pour un montant total prévisionnel de $4190000 €$. 
sibles d'algues planctoniques et de la moule à rayures noires, Mytolopsis sallei, originaire du golfe du Mexique et des Caraïbes.

Pour faire face à cette menace, le PROE, qui est en charge du Programme pour la prévention de la pollution marine dans le Pacifique (PACPOL) a adopté la stratégie régionale sur les espèces envahissantes en 2000. En 2006, il a proposé à ses membres de la compléter par un texte distinct et complémentaire : la « stratégie régionale relative à l'introduction d'organismes marins nuisibles par les navires dans les pays insulaires du Pacifique » (SRIMP-PAC) qui porte sur les vecteurs d'origine maritime (salissures de coques et eaux de ballast). Elle propose également un cadre d'application harmonisée du régime de contrôle et de gestion des organismes marins, en application de la convention internationale pour le contrôle et la gestion des eaux et sédiments de ballast adoptée en février 2004 par l'oмI. La stratégie SRIMP-PAC est organisée sous la forme d'une «défense étagée » inspirée des dispositions adoptées par la Nouvelle-Zélande en matière de sécurité biologique et comprend trois catégories de mesures.

- Avant la frontière : la prévention des introductions

La zone prioritaire est celle du canal de Panama. En effet, un grand nombre de navires (du yacht privé aux grands navires marchands) se concentrent dans une zone restreinte avant de s'engager dans le Pacifique. Une inspection des coques à cet endroit permettrait d'éviter un nombre considérable de bio-invasions marines. Le PROE devrait engager des négociations avec l'État panaméen en vue de la mise en place d'un tel système. En ce qui concerne la gestion des eaux de ballast, il faut cependant signaler qu'aucune technologie pratique et commercialement viable n'existe aujourd'hui en matière de traitement des eaux de ballast à bord d'un navire faisant route.

- À la frontière : l'interdiction des introductions

Elle suppose que les États et territoires du Pacifique soient en mesure de procéder aux examens et visites techniques nécessaires. En ce qui concerne les salissures de coques, seule l'Australie a, à notre connaissance, adopté un protocole de gestion des bio-salissures applicable aux navires appréhendés et aux petits navires effectuant des voyages internationaux. En ce qui concerne la gestion des eaux de ballast, le traitement est relativement plus simple : il suffit que les États et territoires concernés exigent de tous les navires concernés qu'ils présentent un rapport de gestion des eaux de ballast, conformément à la résolution A.868 de l'omi. En cas d'irrégularité, le navire doit repartir vers des eaux profondes (> 200 mètres), pour renouveler ses eaux de ballast. Enfin, concernant les sédiments contenus dans les ballasts, les navires ont bien entendu interdiction de les rejeter dans les ports, mais encore faut-il que le territoire ou l'État océanien ait les moyens de se doter des installations de réception de sédiments.

- En deçà de la frontière : lutte et mesure d'atténuation de l'invasion

Lorsqu'une espèce marine envahissante a été identifiée, il est malheureusement, en règle générale, pratiquement impossible de l'éradiquer, sauf, peut-être, si son implantation est bien circonscrite et de peu d'importance.

\section{Le programme « avenirs océaniens »}

Ce programme vise essentiellement deux domaines : celui de la prévention de la pollution (et notamment la gestion des déchets) et celui des changements climatiques.

- La prévention de la pollution et la gestion des déchets

La totalité des pays et territoires du Pacifique insulaire peuvent faire leur la réflexion désabusée et fataliste - et néanmoins très inquiétante - du gouvernement de Kiribati à la fin des années 1980 :

« Tout ce qui arrive par bateau dans ce pays y reste. »

Le volume, les types et les impacts de déchets ont continué à croître dans les îles océaniennes au point que 2005 fut déclarée par le PROE « année océanienne de lutte contre les déchets ». Contrairement à d'autres initiatives, celle-ci a entraîné certaines réalisations qui peuvent paraître superficielles, sinon dérisoires, pour des Occidentaux mais qui sont significatives pour sensibiliser des Océaniens ${ }^{3}$ aux conséquences de ces pollutions. Ainsi :

- Niue a initié une campagne de nettoyage du bord de ses routes. On peut douter de sa réelle

3. Ou du degré atteint par la perte du souci de la préservation de la nature ? comme elles semblent lointaines, les années 1970 , pendant lesquelles les prêches entendus dans les différentes églises vilipendaient les essais nucléaires français qui « souillaient la terre que Dieu avait offerte aux Océaniens ». 
efficacité et de son impact, quand on constate qu'en Nouvelle-Calédonie cela fait des années que des associations, voire les pouvoirs publics, mettent en place régulièrement des opérations de nettoyage des bords de routes, de la mangrove ou des îlots proches de la côte sans que le volume des collectes diminue pour autant d'une année sur l'autre... En septembre 2008, le quotidien local relevait le succès rencontré par le tri des déchets sur le territoire de l'une des communes proches de Nouméa, mais s'interrogeait sur leur devenir, puisqu'il n'existe pas d'unité de recyclage en Nouvelle-Calédonie...

- En revanche, à Fiji, le slogan « de la monnaie pour vos déchets " a permis de mettre en place des partenariats de recyclage et une campagne de sensibilisation pour aider la population à rentabiliser ses déchets.

- Samoa a entrepris un plan de réduction de l'utilisation des sacs plastiques, visant à leur interdiction et à leur remplacement par des sacs biodégradables constitués à $50 \%$ d'amidon de maïs, ce qui permet leur dégradation en six mois. Parallèlement, une décharge "révolutionnaire » a été inaugurée par les pouvoirs publics : elle substitue à la décharge sauvage un compactage des déchets et une couverture par couche de terre.

- Le Vanuatu s'est fixé la date de 2009 pour réduire de $30 \%$ le volume des déchets mis en décharge, de $50 \%$ les détritus dans les lieux publics et de $80 \%$ l'incinération des déchets à ciel ouvert.

- Tuvalu s'est lancé dans un inventaire des batteries sèches et humides que recèlent les foyers et les entreprises afin d'informer le public de leur dangerosité. Mais, si elles sont un jour collectées, elles devront être acheminées vers l'Australie pour y être retraitées.

L'Australie est, par ailleurs, à l'origine de l'initiative visant à éliminer les polluants organiques persistants (POP), notamment les pesticides insolubles et les polychorobiphényles (РСB) existant dans le Pacifique et contenus essentiellement dans les huiles. Le projet de gestion de ces POP est l'un des tout premiers à être mis en place dans le cadre d'accords internationaux et il contribue à la mise en œuvre de la convention de Stockholm du 22 mai 2001. L'objet est l'élimination des POP dans les treize États océaniens. En 2006, plus de 130 tonnes de POP, dont 60 tonnes d'huiles avaient été traitées ou acheminées vers l'Australie en vue de leur destruction.

- Le nouveau cadre océanien d'intervention sur les changements climatiques

Les changements climatiques et leurs effets restent un domaine prioritaire pour le Pacifique, car certains États océaniens, comme Kiribati ou Tuvalu sont directement menacés de disparition en cas de montée du niveau de la mer ${ }^{4}$. Si le concept de "réfugiés climatiques » devait malheureusement devenir une réalité, les populations de ces deux États en fourniraient sans nul doute les premiers contingents. En 2005, le PROE a donc adopté un nouveau cadre de référence pour la période 2006-2015, afin que les États puissent prendre les mesures les plus appropriées. En matière d'adaptation aux changements climatiques, quatre projets pilotes ont été menés de 2001 à 2005. Ils concernent :

- Le Vanuatu, où la petite communauté du village de Litao a dû être réinstallée dans l'intérieur des terres de Tegua, leur village côtier ayant, à plusieurs reprises, souffert de dégâts causés par des tempêtes et des vagues violentes, mais sans que ces phénomènes puissent être clairement rattachés aux changements climatiques.

- Les îles Cook dont l'alimentation en eau potable s'est dégradée en raison d'une salinisation des réserves d'eau.

- Samoa, où une protection côtière a été érigée pour éviter que la forte érosion des côtes ne détruise pas totalement les terres traditionnelles de la communauté de Saoluafata que plusieurs familles avaient déjà dû quitter.

- Aux îles Fiji, le projet a eu pour but de renforcer la capacité en citernes d'eau afin de faire face aux périodes de sécheresse.

Cependant, il est difficile de mettre en évidence un lien de causalité évident et objectif entre les situations des populations concernées et une éventuelle dégradation des conditions climatiques, les scientifiques étant plus que partagés sur ce point.

Enfin, on notera que, désormais, onze États insulaires participent aux programmes de réduction des émissions de gaz à effet de serre et de protection de la couche d'ozone : les nouveaux participants sont les îles Cook, Nauru et Niue, mais la faiblesse, sinon l'absence de leurs moyens, ne suscite qu'une satisfaction morale.

4. Si Kiribati peut s'enorgueillir de posséder, dans l'île de Banaba, un sommet culminant à 81 mètres d'altitude, le reste de l'archipel a une altitude moyenne de 5 à 10 mètres. Quant à Tuvalu, son point le plus élevé est à 5 mètres au-dessus du niveau de la mer. 
Pour conclure, il serait tentant de souligner que le PROE démontre à merveille la distinction enseignée traditionnellement aux étudiants de droit entre compétence et pouvoir : l'organisation dispose de la compétence nécessaire pour organiser la lutte contre la pollution, mais faute de finances, son pouvoir d'intervention est plus que limité. A'ita péa péa ${ }^{5}$, diront avec insouciance les Polynésiens. Mais pour combien de temps encore?

\section{BIBLIOGRAPHIE}

GreenPeace, 1989. Report on Admiralty Pacific's Proposal to Dispose of US Municipal Garbage in the Marshall Islands, Greenpeace, Washington.

PROE, 2005. Plan d'action pour la gestion de l'environnement dans la région Pacifique : 2005-2009, Samoa, PROE.

—, 2004. Programmes stratégiques du PROE : 20042013, Samoa, PROE.

—, 2004. Rapports annuels, Samoa, PROE.

—, 2005. Rapports annuels, Samoa, PROE.

5. Familièrement, « ce n'est pas trop grave » ou « cela n'a pas trop d'importance ». 
